\title{
A dietary pattern rich in animal organ, seafood and processed meat products is associated with newly diagnosed hyperuricaemia in Chinese adults: a propensity score-matched case-control study
}

\author{
Yang Xia ${ }^{1,2} \dagger$, Qi Xiang ${ }^{3} \dagger$, Yeqing $\mathrm{Gu}^{1} \dagger$, Suwei Jia ${ }^{4}$, Qing Zhang ${ }^{2}$, Li Liu ${ }^{2}$, Ge Meng ${ }^{1}$, Hongmei Wu ${ }^{1}, \mathrm{Xue} \mathrm{Bao}^{1}$, \\ Bin $\mathrm{Yu}^{1}$, Shaomei Sun ${ }^{2}$, Xing $\mathrm{Wang}^{2}$, Ming Zhou ${ }^{2}$, Qiyu Jia ${ }^{2}$, Yuntang Wu ${ }^{1}$, Kun Song ${ }^{2}$ and Kaijun Niu ${ }^{1,2_{*}}$ \\ ${ }^{1}$ Nutritional Epidemiology Institute and School of Public Health, Tianjin Medical University, Tianjin, 300070, People's Republic of China \\ ${ }^{2}$ Health Management Centre, Tianjin Medical University General Hospital, Tianjin, 300070, People's Republic of China \\ ${ }^{3}$ Nutrition Department, Tianjin Nankai Hospital, Tianjin, 300100, People's Republic of China \\ ${ }^{4}$ Tianjin Haibin People Hospital, Tianjin, 300280, People's Republic of China
}

(Submitted 6 October 2017 - Final revision received 29 January 2018 - Accepted 7 March 2018)

\section{Abstract}

Previous studies have indicated that some food items and nutrients are associated with uric acid metabolism in humans. However, little is known about the role of dietary patterns in hyperuricaemia. We designed this case-control study to evaluate the associations between dietary patterns and newly diagnosed hyperuricaemia in Chinese adults. A total of 1422 cases and 1422 controls were generated from 14538 participants using the 1:1 ratio propensity score matching methods. Dietary intake was assessed using a validated self-administered FFQ. Dietary patterns were derived by factor analysis. Hyperuricaemia was defined as concentrations of serum uric acid higher than $7 \mathrm{mg} / \mathrm{dl}(416 \cdot 5 \mu \mathrm{mol} / \mathrm{l})$ for men and $6 \mathrm{mg} / \mathrm{dl}(357 \mu \mathrm{mol} / \mathrm{l})$ for women. Three dietary patterns were derived by factor analysis: sweet pattern; vegetable pattern; animal foods pattern. The animal foods pattern characterised by higher intake of an animal organ, seafood and processed meat products was associated with higher prevalence of newly diagnosed hyperuricaemia $\left(P_{\text {for trend }}<0.01\right)$ after adjustment. Compared with the participants in the lowest quartile of the animal foods pattern, the OR of newly diagnosed hyperuricaemia in the highest quartile was 1.50 (95\% CI 1.20, 1.87). The other two dietary patterns were not associated with the prevalence of newly diagnosed hyperuricaemia after adjustment. In conclusion, a diet rich in animal organ, seafood and processed meat products is associated with higher prevalence of newly diagnosed hyperuricaemia in a Chinese population. Further cohort studies and randomised controlled trials are required to clarify these findings.

\section{Key words: OR: Diets: Dietary patterns: Hyperuricaemia}

Hyperuricaemia is a result of multifactor interactions including sex, age, genetic, environmental factors and diet ${ }^{(1,2)}$. Hyperuricaemia is the major aetiological factor in gout ${ }^{(3)}$ and associated with many other diseases, such as the metabolic syndrome ${ }^{(4)}$, $\mathrm{CVD}^{(5)}$ and kidney diseases ${ }^{(6)}$. A meta-analysis conducted in 2015 suggested that the pooled prevalence of hyperuricaemia is $13.3 \%$ in China ${ }^{(7)}$. Moreover, an increasing trend in the prevalence of hyperuricaemia has been observed over the world in recent years ${ }^{(8,9)}$

Evidence has suggested that diet and nutrition play a significant role in the development of hyperuricaemia ${ }^{(1)}$. Previous studies have demonstrated that some dietary factors are associated with risk of hyperuricaemia, including meat ${ }^{(10)}$, seafood $^{(10)}$, alcohol $^{(11)}$ and sweetened beverages ${ }^{(12)}$. However, regular diets consist of complex combinations of foods and nutrients ingested together that may act independently or may interact with one another. Therefore, examination of dietary patterns, which assess the effects of overall diet, would more closely parallel the real world ${ }^{(13)}$. A cohort study found that higher baseline adherence to the Mediterranean diet is associated with a lower risk of hyperuricaemia ${ }^{(14)}$. However, the analyses were conducted in a sample of elderly Spanish participants at high cardiovascular risk, which would limit the generalisation of their findings. In China, a cross-sectional study with 374 participants demonstrated that the 'animal products and fried foods pattern' (high in pork, eggs, animal giblets, poultry and fried wheat products) is associated with higher prevalence of hyperuricaemia while a 'soyabean products and fruit pattern' (high in soyabean products, fruit, vegetables and starchy tubers) is associated with lower prevalence of hyperuricaemia $^{(15)}$. Another cross-sectional study in Taiwan (266 participants) found that there is no significant association

\footnotetext{
Abbreviation: PA, physical activity.

* Corresponding author: K. Niu, email nkj0809@gmail.com; niukaijun@tmu.edu.cn

$\dagger$ These authors contributed equally to this work.
} 
between dietary patterns and uric acid level in Chinese ${ }^{(16)}$. Considering the small sample size of these two studies, the effect of dietary patterns on hyperuricaemia is still unclear. Moreover, cross-sectional study design may suffer from reverse causation as participants with hyperuricaemia changed their diet to reduce high purine food intake. We thus designed this case-control study with sufficient sample size to provide adequate power to detect the associations between dietary patterns and hyperuricaemia in a general population.

\section{Methods \\ Participants}

This case-control study was based on the Tianjin Chronic Lowgrade Systemic Inflammation and Health Cohort Study, which is a large prospective dynamic cohort study focusing on the relationships between chronic low-grade systemic inflammation and the health status of a population living in Tianjin, China ${ }^{(17)}$. Participants were recruited while having their annual health examinations at the Tianjin Medical University General Hospital-Health Management Center and community management centres in Tianjin. This dynamic cohort study was launched in 2007. Moreover, a detailed lifestyle questionnaire covering family income, marital status, employment status, educational level, physical activity (PA), sleep habits, dietary habits, overall computer/mobile device usage time, television time, history of prior infections and use of medicines as well as physical performance tests were administered to about $70-80 \%$ randomly selected subjects from this population since May 2013. The present study used data of the lifestyle questionnaires of participants collected from May 2013 to December 2016 and data of annual health examinations of participants collected from January 2007 to December 2016.

In all, 23025 participants completed a comprehensive health examination, including evaluation of anthropometric parameters and biochemical blood examination (included fasting blood glucose (FBG), TAG and HDL-cholesterol, etc.). Participants also completed a study questionnaire reporting personal information, dietary intake, lifestyles and health condition. We excluded participants who changed their lifestyles in last 5 years, which including diet, drinking, smoking, PA and sleeping ( $n$ 5875), or those with a history of CVD $(n$ 1051) or cancer ( $n$ 198). We also excluded participants who were diagnosed as hyperuricaemia from January 2007 to May 2013 and those with a history of self-reported hyperuricaemia ( $n$ 1363). The final study population comprised 14538 (1454 cases and 13084 controls) participants for propensity score matching. The protocol of this study was approved by the Institutional Review Board of the Tianjin Medical University and participants gave written informed consent before participation in the study.

\section{Propensity score matching}

The propensity score was calculated using a logistic regression model and the following covariates: sex, age, BMI, PA, energy intake, education level, household income, smoking status, drinking status, employment status and the metabolic syndrome status. Using these propensity scores, cases were individually matched by controls using the nearest matching method within a caliper distance, which selects for matching a control subject whose propensity score is closest to that of the case subject (nearest neighbour matching approach) with the further restriction that the absolute difference in the propensity scores of matched subjects must be below some pre-specified threshold (the caliper distance) ${ }^{(18)}$. Thus, participants for whom the propensity score could not be matched because of a greater caliper distance were excluded from further analysis. As suggested by Austin $^{(18)}$, a caliper of width equal to $0 \cdot 2$ of the standard deviation of the logit of the propensity score was used, as this value minimised the mean squared error of the estimated treatment effect in several scenarios. To better match cases and controls, we used the 1:1 ratio matching method. If a case subject could not be matched to any control subject, then the case subject was discarded. Finally, 1422 cases and 1422 controls were generated using this propensity score matching method.

\section{Identification of dietary patterns}

Dietary intake was assessed using a modified version of the FFQ that included 100 food items (the initial version of the FFQ included eighty-one food items ${ }^{(19)}$ ) with specified serving sizes one time per $2-3$ years. In the present study, we used data of the FFQ filled in by the participants when they first been diagnosed with hyperuricaemia from May 2013 to December 2016. The FFQ included seven frequency categories ranging from 'almost never' to 'twice or more per day' for foods and eight frequency categories ranging from 'almost never' to 'four or more times per day' for beverages in the last month. The mean daily intake of nutrients was calculated by using an ad hoc computer program developed to analyse the questionnaire. The Chinese food composition tables ${ }^{(20)}$ were used as the nutrient database. The reproducibility and validity of the questionnaire were assessed in a random sample of 150 participants from our cohort using data from repeated measurements of the FFQ approximately 3 months apart and 4-d weighed diet records (WDR). Spearman rank correlation coefficient for energy intake between two FFQ administered 3 months apart was $0 \cdot 68$. Correlation coefficients for food items (fruits, vegetables, fish, meat and beverages) between two FFQ administered 3 months apart ranged from 0.62 to 0.79 . Spearman's rank correlation coefficient for energy intake by the WDR and the FFQ was 0.49. Correlation coefficients for nutrients (vitamin C, vitamin E, PUFA, SFA, carbohydrate and Ca) by the WDR and the FFQ ranged from 0.35 to 0.54 . We applied factor analysis in order to generate major dietary patterns and factor loadings on all 100 food items and beverages (g). Varimax rotation was applied for greater interpretability. After evaluation of eigenvalues $(>1 \cdot 0)$ and the scree test, three factors were determined. Food items with a factor loading $>10.301$ were the main contributors to dietary pattern and representative of the character of each pattern. Factors were named descriptively according to the food items showing high loading (absolute value) with respect to each dietary pattern as follows: 'sweet' pattern, 'vegetable' pattern and 'animal foods' pattern. Similar food items were further collapsed into five major food groups based on the characteristics of food items: animal foods, fruits, vegetables, sugared beverages and snacks and high-carbohydrate foods. 


\section{Assessment of hyperuricaemia and other variables}

Hyperuricaemia was defined as concentrations of serum uric acid higher than $7 \mathrm{mg} / \mathrm{dl}(416.5 \mu \mathrm{mol} / \mathrm{l})$ in men and higher than $6 \mathrm{mg} / \mathrm{dl}(357 \mu \mathrm{mol} / \mathrm{l})$ in women ${ }^{(21)}$.

Waist circumference was measured at the umbilical level with participants standing and breathing normally. Blood pressure (BP) was measured twice from the upper left arm using a TM-2655P automatic device (A\&D Co.) after 5 min of rest in a seated position. The mean of these two measurements was taken as the BP value. Blood samples for the analysis of FBG and lipids were collected in siliconised vacuum plastic tubes. FBS was measured by the glucose oxidase method, TAG were measured by enzymatic methods, LDL-cholesterol was measured by the polyvinyl sulphuric acid precipitation method, and HDL-cholesterol was measured by the chemical precipitation method using reagents from Roche Diagnostics on an automatic biochemistry analyzer (Cobas 8000 modular analyzer; Roche). The metabolic syndrome was defined in accordance with the criteria for China of the American Heart Association and International Diabetes Federation scientific statement of $2009^{(22)}$. Participants were considered to have the metabolic syndrome when they presented three or more of the following components: (1) elevated waist circumference for Chinese individuals ( $\geq 85 \mathrm{~cm}$ in males; $\geq 80 \mathrm{~cm}$ in females), (2) elevated TAG $(\geq 1.7 \mathrm{mmol} / \mathrm{l})$, or drug treatment for elevated TAG, (3) reduced $\mathrm{HDL}(<1.0 \mathrm{mmol} / \mathrm{l}$ in males; $<1.3 \mathrm{mmol} / 1$ in females) or drug treatment for reduced HDL, (4) elevated BP (systolic BP $\geq 130 \mathrm{~mm} \mathrm{Hg}$ and/or diastolic BP $\geq 85 \mathrm{~mm} \mathrm{Hg}$ ) or antihypertensive drug treatment, (5) elevated fasting glucose $(\geq 5.56 \mathrm{mmol} / \mathrm{l})$ or drug treatment for elevated glucose

The socio-demographic variables, which include sex, age, education, employment and household income, were also assessed by questionnaire. The educational level was assessed by asking the question 'what is the highest degree you earned?' and was divided into two categories: <college graduate or $\geq$ college graduate. Employment status was classified as either senior officials and managers or professionals. Information on the smoking ('never', 'former', and 'current smoking') and drinking ('never', 'former', and 'current drinking') status of the participants was obtained from a questionnaire survey. Participants who changed their lifestyles, including diet, drinking, smoking, activity and sleeping was assessed by asking the question 'have you ever changed your lifestyles (including diet, drinking, smoking, PA and sleeping) in last 5 years?' and was divided into two categories: yes or no. PA in the most recent week was assessed using the short form of the International Physical Activity Questionnaire ${ }^{(23)}$. The questionnaire asked whether subjects had performed any activities from the following categories during the previous week: walking; moderate activity (household activity or child care); vigorous activity (running, swimming or other sports activities). Metabolic equivalent (MET)-hours per week were calculated using corresponding MET coefficients (3.3, $4 \cdot 0$ and 8.0, respectively) according to the following formula: MET coefficient of activity $\times$ duration $(\mathrm{h}) \times$ frequency $(\mathrm{d})$. Total PA levels were assessed by combining separate scores for different activities.

\section{Statistical analysis}

In order to characterise participants according to newly diagnosed hyperuricaemia status, descriptive data have been presented as the least-square means and 95\% CI or as percentages and examined using ANOVA and $\chi^{2}$ test for categorical variables. Quartiles were categorised across the scores of each dietary pattern based on the distribution of the scores for all the participants and used for further analyses. Association between quartilecategories of dietary pattern scores and hyperuricaemia status were examined using conditional logistic regression analysis. Newly diagnosed hyperuricaemia status was used as dependent variable, and factor score was used as an independent variable. OR and 95\% CI were calculated. The linear trend cross increasing quartiles was tested using the median value of each quartile as a continuous variable based on linear regression. Model 1 was used to calculate the crude OR and model 2 additionally adjusted for scores of other dietary patterns or intake of other food groups with each other. All analyses were performed using the Statistical Analysis System 9.3 edition for Windows (SAS Institute Inc.) and STATA (version 12.1; StataCorp LP). All $P$ values were two-tailed and the difference was defined to be significant when $P<0.05$.

\section{Results}

\section{Dietary patterns}

Factor analysis revealed three main dietary patterns (Table 1), which accounted for $22.6 \%$ of the variance in total food intake. According to the contribution to the total variance, the three dietary patterns were: factor 1 was defined as the sweet pattern and characterised by high intake of candied fruits, cakes and ice cream; factor 2 , the vegetable pattern, was typified by intake of vegetables, soya products and coarse cereals; factor 3, identified as the animal foods pattern and included intake of animal organ, seafood and processed meat products.

\section{Characteristics of participants}

Characteristics of participants according to newly diagnosed hyperuricaemia status before and after propensity scores matching are shown in Tables 2 and 3, respectively.

Among 14538 participants who were available to be analysed before propensity score matching, 10\% were classified as newly diagnosed hyperuricaemia. As shown in Table 2, participants with newly diagnosed hyperuricaemia trended to be men $(P<0 \cdot 0001)$, younger $(P<0.0001)$, current smoker $(P<0.0001)$, ex-smoker $(P=0.02)$, drinking everyday $(P<0.0001)$ or sometimes $(P<0 \cdot 0001)$, who had higher BMI $(P<0.0001)$, higher energy intake $(P<0.01)$ and higher prevalence of the metabolic syndrome $(P<0 \cdot 0001)$. After propensity score matching, 1422 cases and 1422 controls were generated and showed no significant differences in any character (Table 3).

\section{Dietary patterns and hyperuricaemia}

As shown in Table 4, higher adherence to the animal foods pattern was associated with higher prevalence of newly diagnosed hyperuricaemia before and after adjustment for the 
Table 1. The factor loadings scores* of primary food groups of dietary patterns

\begin{tabular}{|c|c|c|c|c|c|}
\hline \multicolumn{2}{|l|}{ Sweet pattern } & \multicolumn{2}{|l|}{ Vegetable pattern } & \multicolumn{2}{|l|}{ Animal foods pattern } \\
\hline Food items & $\begin{array}{l}\text { Factor } \\
\text { loadings }\end{array}$ & Food items & $\begin{array}{l}\text { Factor } \\
\text { loadings }\end{array}$ & Food items & $\begin{array}{l}\text { Factor } \\
\text { loadings }\end{array}$ \\
\hline Strawberry, kiwi fruit, persimmon & 0.72 & Cucumber & 0.63 & Animal organ (except for animal liver) & 0.60 \\
\hline Sweets, candied fruits & 0.69 & Chinese cabbage & 0.62 & Animal blood & 0.58 \\
\hline Cookies & 0.68 & Celery & 0.58 & Animal liver & 0.55 \\
\hline Chinese cakes & 0.68 & Green vegetable & 0.56 & Preserved egg & 0.54 \\
\hline Salted eggs & 0.67 & Eggplant & 0.55 & Seafood (shellfish, squid, shrimp) & 0.49 \\
\hline Preserved bean curd & 0.67 & Pumpkin, carrot & 0.52 & Sausage & 0.48 \\
\hline Western-style pastry, cakes & 0.66 & Chinese watermelon & 0.52 & Freshwater fish & 0.47 \\
\hline Ice cream & 0.66 & Raw vegetables (except for tomato) & 0.50 & Sea fish & 0.43 \\
\hline Chinese sauerkraut & 0.65 & Coarse cereals & 0.38 & White wine & 0.40 \\
\hline Pineapple & 0.64 & Tomato (including the ketchup) & 0.38 & Red wine & 0.38 \\
\hline Leek & 0.63 & Congee & 0.36 & Pork skin & 0.37 \\
\hline Sea-plant & 0.62 & Potato (except for sweet potato) & 0.36 & Carbonated beverage & 0.34 \\
\hline Onion & 0.61 & Radish (expect for carrot) & 0.35 & Beer & 0.33 \\
\hline Pear & 0.60 & Soyabean products & 0.33 & Fruit juice, vegetable juice & 0.32 \\
\hline Walnut & 0.60 & Egg & 0.33 & Steamed stuffed bun, dumpling & 0.31 \\
\hline Peach & 0.60 & Mushroom & 0.31 & Miscellaneous sauce noodles & 0.30 \\
\hline Lotus root & 0.59 & Sweet potato & 0.30 & Wonton & 0.29 \\
\hline Other kinds of fruit & 0.57 & Freshwater fish & 0.30 & Chinese watermelon & 0.29 \\
\hline Mushroom & 0.56 & Apple & 0.29 & Instant noodle & 0.29 \\
\hline Other types of beans & 0.56 & Bell peppers & 0.28 & Coffee & 0.28 \\
\hline
\end{tabular}

${ }^{*}$ For simplicity, only the top twenty food groups of factor loading scores of each pattern are shown.

Table 2. Participant characteristics by hyperuricaemia status before matching (Least-square means and $95 \%$ confidence intervals)

\begin{tabular}{|c|c|c|c|c|c|}
\hline \multirow[b]{3}{*}{ Characteristics } & \multicolumn{4}{|c|}{ Hyperuricaemia status (before matching) } & \multirow[b]{3}{*}{$P^{*}$} \\
\hline & \multicolumn{2}{|c|}{ No $(n$ 13084) } & \multicolumn{2}{|c|}{ Yes $(n$ 1454) } & \\
\hline & Mean & $95 \% \mathrm{Cl}$ & Mean & $95 \% \mathrm{Cl}$ & \\
\hline Sex (male \%) & \multicolumn{2}{|r|}{$47 \cdot 31$} & \multicolumn{2}{|r|}{$80 \cdot 33$} & $<0.0001$ \\
\hline Age (years) & 42.61 & $42 \cdot 41,42 \cdot 82$ & 40.41 & $39.84,40.99$ & $<0.0001$ \\
\hline BMI $\left(\mathrm{kg} / \mathrm{m}^{2}\right)$ & 23.88 & $23.82,23.94$ & $26 \cdot 98$ & $26 \cdot 78,27 \cdot 18$ & $<0.0001$ \\
\hline Physical activity (Mets $\times \mathrm{h} /$ week) & 9.39 & $9.17,9.61$ & 10.00 & $9.32,10.73$ & $0 \cdot 10$ \\
\hline Energy intake (kJ/d) & 8313.23 & $8270 \cdot 82,8355 \cdot 84$ & $8537 \cdot 21$ & $8407 \cdot 24,8669 \cdot 19$ & $<0.01$ \\
\hline Education ( $\geq$ college graduate, $\%$ ) & & 60.72 & & 59.93 & 0.56 \\
\hline Household income ( $\geq 10000$ Yuan, \%) & & 34.70 & & $36 \cdot 31$ & 0.22 \\
\hline Metabolic syndrome (yes, \%) & & 21.49 & & 51.58 & $<0.0001$ \\
\hline \multicolumn{6}{|l|}{ Smoking status $(\%)$} \\
\hline Smoker & & $19 \cdot 27$ & & 34.03 & $<0.0001$ \\
\hline Ex-smoker & & 4.70 & & $6 \cdot 21$ & 0.02 \\
\hline Non-smoker & & $76 \cdot 03$ & & 59.76 & $<0.0001$ \\
\hline \multicolumn{6}{|l|}{ Drinking status (\%) } \\
\hline Everyday & & $5 \cdot 17$ & & $9 \cdot 14$ & $<0.0001$ \\
\hline Sometime & & 54.75 & & 63.57 & $<0.0001$ \\
\hline Ex-drinker & & $8 \cdot 27$ & & 9.00 & 0.34 \\
\hline Non-drinker & & $31 \cdot 81$ & & $18 \cdot 28$ & $<0.0001$ \\
\hline \multicolumn{6}{|l|}{ Employment status (\%) } \\
\hline Managers & & $43 \cdot 10$ & & 40.85 & $0 \cdot 11$ \\
\hline Professionals & & $16 \cdot 78$ & & $16 \cdot 75$ & 0.98 \\
\hline Others & & $40 \cdot 13$ & & 42.40 & 0.11 \\
\hline
\end{tabular}

${ }^{*}$ ANOVA or $x^{2}$ test.

scores of other two dietary patterns $\left(P_{\text {for trend }}<0 \cdot 01\right)$. Compared with the participants in the lowest quartile, the OR for the extreme quartile is 1.51 (95\% CI 1.21, 1.87) and 1.50 (95\% CI $1 \cdot 20,1.87)$ before and after adjustment, respectively. The other two dietary patterns were not associated with the prevalence of newly diagnosed hyperuricaemia after adjustment.

Results of major food groups' analyses are presented in Table 5. Consumption of animal foods was associated with a higher prevalence of newly diagnosed hyperuricaemia ( $P_{\text {for }}$ trend $<0.001$ ) after adjustment for intake of other food groups. Compared with the participants in the lowest quartile, the OR for the extreme quartile was $1.48(95 \%$ CI 1.18, 1.85). We also found a high intake of fruits was associated with a higher prevalence of newly diagnosed hyperuricaemia after adjustment, the OR for the participants in the extreme quartile was 1.34 (95\% CI 1.07, 1.69) 
Table 3. Participant characteristics by hyperuricaemia status after matching (Least-square means and $95 \%$ confidence intervals)

\begin{tabular}{|c|c|c|c|c|c|}
\hline \multirow[b]{3}{*}{ Characteristics } & \multicolumn{4}{|c|}{ Hyperuricaemia status (after matching) } & \multirow[b]{3}{*}{$P^{*}$} \\
\hline & \multicolumn{2}{|c|}{ No $(n$ 1422) } & \multicolumn{2}{|c|}{ Yes ( $n$ 1422) } & \\
\hline & Mean & $95 \% \mathrm{Cl}$ & Mean & $95 \% \mathrm{Cl}$ & \\
\hline Sex (male \%) & \multicolumn{2}{|r|}{79.96} & \multicolumn{2}{|r|}{79.89} & 0.96 \\
\hline Age (years) & $40 \cdot 85$ & $40 \cdot 26,41 \cdot 44$ & $40 \cdot 58$ & $39.99,41 \cdot 17$ & 0.53 \\
\hline BMI $\left(\mathrm{kg} / \mathrm{m}^{2}\right)$ & $26 \cdot 89$ & $26 \cdot 71,27 \cdot 09$ & $26 \cdot 84$ & $26 \cdot 65,27 \cdot 03$ & 0.69 \\
\hline Physical activity (Mets $\times$ hours/week) & $9 \cdot 89$ & $9 \cdot 21,10 \cdot 61$ & $10 \cdot 00$ & $9.31,10.73$ & 0.82 \\
\hline Energy intake (kJ/d) & $8568 \cdot 27$ & $8442 \cdot 78,8695 \cdot 60$ & 8522.06 & $8397 \cdot 24,8648 \cdot 72$ & 0.61 \\
\hline Education ( $\geq$ college graduate, $\%$ ) & & $60 \cdot 27$ & & $60 \cdot 16$ & 0.95 \\
\hline Household income ( $\geq 10000$ Yuan, \%) & & $37 \cdot 23$ & & $36 \cdot 16$ & 0.56 \\
\hline Metabolic syndrome (yes, \%) & & $50 \cdot 42$ & & $50 \cdot 56$ & 0.94 \\
\hline \multicolumn{6}{|l|}{ Smoking status (\%) } \\
\hline Smoker & & 34.49 & & 33.88 & 0.75 \\
\hline Ex-smoker & & $6 \cdot 85$ & & $6 \cdot 29$ & 0.57 \\
\hline Non-smoker & & 58.66 & & 59.84 & 0.55 \\
\hline \multicolumn{6}{|l|}{ Drinking status (\%) } \\
\hline Everyday & & $9 \cdot 18$ & & 8.99 & 0.86 \\
\hline Sometime & & 62.99 & & 63.74 & 0.68 \\
\hline Ex-drinker & & 7.97 & & 8.92 & 0.36 \\
\hline Non-drinker & & $19 \cdot 86$ & & $18 \cdot 34$ & 0.31 \\
\hline \multicolumn{6}{|l|}{ Employment status (\%) } \\
\hline Managers & & 42.49 & & 41.09 & 0.46 \\
\hline Professionals & & 14.49 & & $16 \cdot 98$ & 0.08 \\
\hline Others & & 43.02 & & 41.93 & 0.57 \\
\hline
\end{tabular}

* ANOVA or $x^{2}$ test.

Table 4. Association between quartiles of factor scores and hyperuricaemia (Odds ratios and $95 \%$ confidence intervals)

\begin{tabular}{|c|c|c|c|c|c|c|c|c|}
\hline \multirow[b]{2}{*}{ Dietary patterns } & \multicolumn{8}{|c|}{ Quartiles of factor scores (range, $n$ 2844) } \\
\hline & & OR & $95 \% \mathrm{Cl}$ & OR & $95 \% \mathrm{Cl}$ & OR & $95 \% \mathrm{Cl}$ & $P_{\text {for trend }}{ }^{*}$ \\
\hline Sweet pattern & Level $1(-2.68,-0.41)$ & \multicolumn{2}{|c|}{ Level $2(-0.41,-0.17)$} & \multicolumn{2}{|c|}{ Level $3(-0 \cdot 17,0 \cdot 15)$} & \multicolumn{2}{|c|}{ Level $4(0.15,11.57)$} & \\
\hline No. of hyperuricemia & 351 & \multicolumn{2}{|c|}{353} & \multicolumn{2}{|c|}{354} & \multicolumn{2}{|c|}{364} & \\
\hline Crude & Ref. & 1.01 & $0.82,1.25$ & 1.02 & $0.83,1.25$ & 1.08 & $0.87,1.33$ & 0.48 \\
\hline Adjusted $†$ & Ref. & 1.03 & $0.83,1.27$ & 1.04 & $0.85,1.28$ & $1 \cdot 10$ & $0.89,1.37$ & 0.36 \\
\hline Vegetable pattern & Level $1(-4 \cdot 75,-0.64)$ & \multicolumn{2}{|c|}{ Level $2(-0.64,-0.15)$} & \multicolumn{2}{|c|}{ Level $3(-0.15,0.41)$} & \multicolumn{2}{|c|}{ Level $4(0.41,7 \cdot 70)$} & \\
\hline No. of hyperuricemia & 368 & \multicolumn{2}{|c|}{360} & \multicolumn{2}{|c|}{350} & \multicolumn{2}{|c|}{344} & \\
\hline Crude & Ref. & 0.96 & $0.78,1.18$ & 0.91 & $0.74,1.11$ & 0.87 & $0.71,1.08$ & 0.17 \\
\hline Adjusted $†$ & Ref. & 0.98 & $0.80,1 \cdot 21$ & 0.92 & $0.76,1 \cdot 13$ & 0.88 & $0.71,1.09$ & 0.19 \\
\hline Animal foods pattern & Level $1(-3.50,-0.54)$ & \multicolumn{2}{|c|}{ Level $2(-0.54,-0.21)$} & \multicolumn{2}{|c|}{ Level $3(-0.21,0.28)$} & \multicolumn{2}{|c|}{ Level $4(0.28,11.77)$} & \\
\hline No. of hyperuricemia & 322 & & 366 & & 345 & & 389 & \\
\hline Crude & Ref. & $1 \cdot 30$ & $1.05,1.60$ & $1 \cdot 16$ & $0.94,1.43$ & 1.51 & $1 \cdot 21,1 \cdot 87$ & $<0.01$ \\
\hline Adjusted† & Ref. & $1 \cdot 30$ & $1.05,1.60$ & $1 \cdot 15$ & $0.93,1.43$ & 1.50 & $1 \cdot 20,1 \cdot 87$ & $<0.01$ \\
\hline
\end{tabular}

Ref., referent values.

* Multiple logistic regression analysis.

† Adjusted for the scores of other two dietary patterns.

( $\left.P_{\text {for trend }}<0 \cdot 01\right)$ when compared with the lowest quartile. Consumption of sugared beverages/snacks $\left(P_{\text {for trend }}=0.03\right)$ was positively associated with newly diagnosed hyperuricaemia after adjustment.

\section{Discussion}

In this study, three main dietary patterns were identified among Chinese adults; the sweet pattern, the vegetable pattern and the animal foods pattern. The main finding of this case-control study is that the animal foods pattern is associated with higher prevalence of newly diagnosed hyperuricaemia in Chinese adults. This association is independent of confounding factors, such as socio-demographic, behavioural, metabolic, health status factors and consumption of other dietary patterns.

A cohort study conducted in a sample of elderly Spanish participants at high cardiovascular risk found that higher baseline adherence to the Mediterranean diet is associated with a lower risk of hyperuricaemia ${ }^{(14)}$. However, these findings cannot be generalised to the general population. Moreover, another limitation is the possibility of reverse causation bias, as high-risk patients may be more likely to change their food habits as a consequence of these conditions being diagnosed. A previous case-control study conducted in 374 Chinese participants 
Table 5. Association between quartiles of intake of major food groups and hyperuricaemia (Odds ratios and $95 \%$ confidence intervals)

\begin{tabular}{|c|c|c|c|c|c|c|c|c|}
\hline \multirow[b]{2}{*}{ Food groups } & & \multicolumn{6}{|c|}{ Quartiles of food group intake (g) ( $n$ 2844) } & \multirow[b]{2}{*}{$P_{\text {for trend }}{ }^{*}$} \\
\hline & & OR & $95 \% \mathrm{Cl}$ & OR & $95 \% \mathrm{Cl}$ & OR & $95 \% \mathrm{Cl}$ & \\
\hline Animal foods & Level $1(0,116)$ & \multicolumn{2}{|c|}{ Level $2(116,163)$} & \multicolumn{2}{|c|}{ Level $3(163,227)$} & \multicolumn{2}{|c|}{ Level $4(227,1183)$} & \\
\hline No. of hyperuricaemia & 320 & \multicolumn{2}{|r|}{352} & \multicolumn{2}{|r|}{366} & \multicolumn{2}{|c|}{384} & \\
\hline Crude & Ref. & 0.94 & $0.77,1.16$ & 1.04 & $0.85,1.28$ & 1.35 & $1.09,1.66$ & $<0.01$ \\
\hline Adjustedt & Ref. & 0.97 & $0.78,1.20$ & 1.09 & $0.88,1.35$ & 1.48 & $1.18,1.85$ & $<0.001$ \\
\hline Fruits & Level $1(0,174)$ & \multicolumn{2}{|c|}{ Level $2(174,295)$} & \multicolumn{2}{|c|}{ Level $3(295,488)$} & \multicolumn{2}{|c|}{ Level $4(488,3440)$} & \\
\hline No. of hyperuricaemia & 350 & \multicolumn{2}{|r|}{324} & \multicolumn{2}{|r|}{361} & \multicolumn{2}{|c|}{387} & \\
\hline Crude & Ref. & 0.97 & $0.79,1.19$ & 1.04 & $0.84,1.28$ & 1.25 & $1.02,1.54$ & 0.02 \\
\hline Adjusted $\dagger$ & Ref. & 0.99 & $0.80,1.22$ & 1.08 & $0.87,1.34$ & 1.34 & $1.07,1.69$ & $<0.01$ \\
\hline Vegetables & Level $1(0,177)$ & \multicolumn{2}{|c|}{ Level $2(177,251)$} & \multicolumn{2}{|c|}{ Level $3(251,355)$} & \multicolumn{2}{|c|}{ Level $4(355,2190)$} & \\
\hline No. of hyperuricaemia & 363 & \multicolumn{2}{|r|}{349} & \multicolumn{2}{|c|}{356} & \multicolumn{2}{|c|}{354} & \\
\hline Crude & Ref. & 1.23 & $1.00,1.52$ & 0.94 & $0.76,1.15$ & 1.06 & $0.86,1.31$ & 0.75 \\
\hline Adjusted $\dagger$ & Ref. & 1.19 & $0.96,1.47$ & 0.89 & $0.72,1.10$ & 0.91 & $0.72,1.16$ & 0.12 \\
\hline Sugared beverages and snacks & Level $1(0,16)$ & \multicolumn{2}{|c|}{ Level $2(16,57)$} & \multicolumn{2}{|c|}{ Level $3(57,137)$} & \multicolumn{2}{|c|}{ Level $4(137,2183)$} & \\
\hline No. of hyperuricaemia & 349 & \multicolumn{2}{|c|}{320} & \multicolumn{2}{|c|}{360} & \multicolumn{2}{|c|}{393} & \\
\hline Crude & Ref. & 0.96 & $0.78,1.18$ & 1.14 & $0.92,1.40$ & 1.30 & $1.06,1.59$ & $<0.01$ \\
\hline Adjusted $\dagger$ & Ref. & 0.93 & $0.75,1.14$ & $1 \cdot 10$ & $0.89,1.35$ & $1 \cdot 20$ & $0.96,1.50$ & 0.03 \\
\hline High-carbohydrate foods & Level $1(0,371)$ & \multicolumn{2}{|c|}{ Level $2(371,503)$} & \multicolumn{2}{|c|}{ Level $3(503,669)$} & \multicolumn{2}{|c|}{ Level $4(669,2620)$} & \\
\hline No. of hyperuricaemia & 349 & \multicolumn{2}{|r|}{353} & \multicolumn{2}{|r|}{364} & \multicolumn{2}{|c|}{356} & \\
\hline Crude & Ref. & 1.19 & $0.97,1.47$ & 0.98 & $0.80,1.21$ & 1.09 & $0.88,1.34$ & 0.88 \\
\hline Adjusted $\dagger$ & Ref. & 1.17 & $0.95,1.45$ & 0.97 & $0.78,1.20$ & 1.05 & $0.84,1.30$ & 0.84 \\
\hline
\end{tabular}

Ref., referent values.

* Multiple logistic regression analysis.

$†$ Adjusted for intake of other food groups.

demonstrated that a dietary pattern rich in pork, eggs, animal giblets, poultry and fried wheat products is positively associated with the prevalence of hyperuricaemia (OR 2.15; 95\% CI 1.22 , 3.76). Meanwhile, a dietary pattern rich in soyabean products, fruit, vegetables and starchy tubers is negatively associated with the prevalence of hyperuricaemia (OR 0.32; 95\% CI 0.19, $0 \cdot 57)^{(15)}$. However, the FFQ in this study includes thirty-three food items (for analyses, the thirty-three food items were collapsed into twenty predefined food groups) ${ }^{(15)}$. Recommendations based on ratios of a sample size to a number of parameters suggested that twenty observations per parameter can uncover the proper factor structure and estimate the loading without bias ${ }^{(24)}$. Thus, the small sample size in this study may affect the accuracy and stability of the conclusion. Another study conducted in Taiwan (266 participants) suggested that there is no significant association between any dietary pattern and hyperuricaemia after adjustment ${ }^{(16)}$. The FFQ in this study has thirty food items and were aggregated into thirteen food groups according to similar macronutrient intakes and eating habits. Thus, the sample size (266 participants and thirteen food groups) was barely enough to perform the factor analysis. However, this procedure resulted in less detailed of intake information. Meanwhile, the adjustment in this study was incomplete (i.e. the metabolic syndrome status was not adjusted). Moreover, the participants who have changed their lifestyles (such as lifestyles of diet, drinking, smoking and activity) in recent years were not been excluded in these studies which could affect the associations between dietary patterns and hyperuricaemia. We thus designed this case-control study with sufficient sample size to provide adequate power to detect the associations between dietary patterns and newly diagnosed hyperuricaemia in a general population who have not changed their lifestyles in last 5 years.
We observed a positive association between consumption of the animal foods pattern, which is typified by intake of animal organ, seafood and processed meat products, and the prevalence of newly diagnosed hyperuricaemia after adjustment of the scores of other two dietary patterns $\left(P_{\text {for trend }}<0 \cdot 01\right)$. This result confirms a previous study which found that 'animal products and fried food' pattern score is associated with a higher prevalence of hyperuricaemia even though that study may have potential inaccurate results due to small ratios of sample size to number of parameters as mentioned before ${ }^{(15)}$. The mechanisms that have been proposed to explain this association is major related the high purine content of animal foods. The suspicion that there is a link between purine-rich diets and hyperuricaemia has been based on metabolic experiments in animals and humans that examined the effect of the artificial short-term loading of purified purine on the serum urate level $^{(25)}$. A previous study demonstrated that compared with those in the lowest quintile, individuals in the highest quintile of purine-rich foods intake, such as meat (RR 1.41;95\% CI 1.07, 1.86) and seafood (RR 1.51; $95 \%$ CI 1.17, 1.95), are associated with an increased risk of gout ${ }^{(10)}$. The major food groups' analyses confirm this finding. In lines with previous studies $^{(10,26)}$, in the analyses of major food groups, our results suggest that animal foods $\left(P_{\text {for trend }}<0 \cdot 001\right)$ intake is associated with the prevalence of newly diagnosed hyperuricaemia after adjustment.

We also found a high intake of fruits is positively associated with newly diagnosed hyperuricaemia after adjustment ( $P_{\text {for trend }}<0.01$ ) in the major food groups' analyses. Compared with the participants in the lowest quartile, the OR for the extreme quartile is 1.34 (95\% CI 1.07, 1.69). A previous study demonstrated that fructose could increase uric acid level ${ }^{(27)}$ 
even though fruits contain lots of vitamin $\mathrm{C}$ and dietary fibres and then benefit to hyperuricaemia. A prospective cohort study suggested that fructose-rich fruits are associated with a higher risk of gout $\left(P_{\text {for trend }}<0 \cdot 05\right)^{(28)}$. More work is needed to understand the dose-response relationship between fruit intake and hyperuricaemia. In line with previous studies ${ }^{(27,29,30)}$, we also found a positive association between sugared beverages/ snacks intake and the prevalence of newly diagnosed hyperuricaemia $\left(P_{\text {for trend }}=0.03\right)$. The mechanisms that have been proposed to explain this association is major related to the fructose content of these kinds of food ${ }^{(27)}$. Moreover, higher consumption of sugared beverage/snacks may represent unmeasured certain lifestyle in the present study that may lead to a higher prevalence of hyperuricaemia. Considering that the main purpose of the present study is to explore the associations between dietary patterns and the prevalence of hyperuricaemia, we will explore more on the associations between sugared beverages/snacks and the prevalence of hyperuricaemia in further studies.

The strengths of our study include our inclusion of participants who were newly diagnosed with hyperuricaemia and our exclusion of participants who had changed their lifestyles in last 5 years. Thus, the reverse causation (i.e. participants with hyperuricaemia changing their diet to reduce high purine food intake) has been corrected as much as possible. Moreover, propensity scores matching methods are being increasingly used to offer investigators the ability to balance treated groups across all putative risk factors. These methods also allow for easy inspection of the balance across measured covariates ${ }^{(31)}$. In this study, the propensity scores matching approach reduced the differences between the case group and the control group. Thus, the associations between dietary patterns and hyperuricaemia were independent of confounding factors.

The present study has some limitations. First, due to the nature of the self-reporting questionnaire, recall bias existed and the food intake may not be exact. Second, we excluded participants for reason of health conditions and the final sample may not be representative of the population. However, it is reasonable to exclude these participants with CVD and cancer when we estimate the association between dietary patterns and hyperuricaemia. Third, we cannot rule out the possibility that unmeasured factors might contribute to the association observed. Finally, the three dietary patterns identified in the present study accounted for $22.6 \%$ of the overall variance, which is slightly lower than other studies ${ }^{(15,19)}$. Aggregating similar food items into one food group is a way to improve variance of dietary patterns but resulted in less detailed intake information. Considering that we did not want to lose any detailed information regarding diet, we used all 100 food items to conduct factor analyses in the present study. Even though it would lead to less total variance of dietary patterns, it would provide more detailed information about dietary patterns.

\section{Conclusions}

In conclusion, in this study, higher scores for a diet rich in animal organ, seafood and processed meat products is associated with higher prevalence of newly diagnosed hyperuricaemia. Further cohort studies and randomised controlled trials are required to clarify these findings.

\section{Acknowledgements}

The authors gratefully thank all of the participants in the study and Tianjin Medical University General Hospital-Health Management Center for the opportunity to perform the study.

This study was supported by grants from the National Natural Science Foundation of China (no. 81673166, 81372118, 81372467 and 81302422), the key technologies R\&D program of Tianjin (Key Project: no. 11ZCGYSY05700, 12ZCZDSY20400, 13ZCZDSY20200, and 15YFYZSY00020), the National Science and Technology Support Program (no. 2012BAI02B02), 2012 and 2016 Chinese Nutrition Society (CNS) Nutrition Research Foundation - DSM Research Fund (no. 2014-071, 2016-046 and 2016-023), the Technologies development program of Beichen District of Tianjin (no. bcws2013-21, bcws2014-05 and 2015SHGY-02), the technologies project of Tianjin Binhai New Area (no. 2013-02-04 and 2013-02-06), the Science Foundation of Tianjin Medical University (no. 2010KY28 and 2013KYQ24), the Key Laboratory of Public Health Safety (Fudan University), Ministry of Education (no. GW2014-5), and the National Training Programs of Innovation and Entrepreneurship for Undergraduates (no. 201510062013), China.

Y. X. and K. N. contributed to the study conception and design; Y. X., G. M., Q. Z., H. W., L. L., Y. G., X. B. and B. Y. contributed to data collection, assembly, analysis and interpretation of the data; S. S., X. W., M. Z., Q. J., Y. W. and K. S. contributed to data collection; Q. X. and S. J. contributed to the revising of the manuscript; Y. X. and K. N., contributed to the manuscript drafting and approval of the final version of the manuscript.

The authors declare that there are no conflicts of interest.

\section{References}

1. Choi HK (2010) A prescription for lifestyle change in patients with hyperuricemia and gout. Curr Opin Rheumatol 22, 165-172.

2. B L, T W, Hn Z, et al. (2011) The prevalence of hyperuricemia in China: a meta-analysis. BMC Public Health 11, 832.

3. Doghramji PP \& Wortmann RL (2012) Hyperuricemia and gout: new concepts in diagnosis and management. Postgrad Med 124, 98-109.

4. Billiet L, Doaty S, Katz JD, et al. (2014) Review of hyperuricemia as new marker for metabolic syndrome. ISRN Rheumatol 2014, 852954.

5. Borghi C, Verardi FM, Pareo I, et al. (2014) Hyperuricemia and cardiovascular disease risk. Expert Rev Cardiovasc Ther 12, 1219-1225.

6. Isaka Y, Takabatake Y, Takahashi A, et al. (2016) Hyperuricemia-induced inflammasome and kidney diseases. Nephrol Dial Transplant 31, 890-896.

7. Liu R, Han C, Wu D, et al. (2015) Prevalence of Hyperuricemia and Gout in Mainland China from 2000 to 2014: a systematic review and meta-analysis. Biomed Res Int 2015, 762820.

8. Zhu Y, Pandya BJ \& Choi HK (2011) Prevalence of gout and hyperuricemia in the US general population: the National Health and Nutrition Examination Survey 2007-2008. Arthritis Rheum 63, 3136-3141. 
9. Roddy E \& Choi HK (2014) Epidemiology of gout. Rheum Dis Clin North Am 40, 155-175.

10. Choi HK, Atkinson K, Karlson EW, et al. (2004) Purine-rich foods, dairy and protein intake, and the risk of gout in men. N Engl J Med 350, 1093-1103.

11. Choi HK, Atkinson K, Karlson EW, et al. (2004) Alcohol intake and risk of incident gout in men: a prospective study. Lancet 363, 1277-1281.

12. Gao X, Qi L, Qiao N, et al. (2007) Intake of added sugar and sugar-sweetened drink and serum uric acid concentration in US men and women. Hypertension 50, 306-312.

13. Hu FB (2002) Dietary pattern analysis: a new direction in nutritional epidemiology. Curr Opin Lipidol 13, 3-9.

14. Guasch-Ferre M, Bullo M, Babio N, et al. (2013) Mediterranean diet and risk of hyperuricemia in elderly participants at high cardiovascular risk. J Gerontol A Biol Sci Med Sci 68, 1263-1270.

15. Zhang M, Chang H, Gao Y, et al. (2012) Major dietary patterns and risk of asymptomatic hyperuricemia in Chinese adults. J Nutr Sci Vitaminol (Tokyo) 58, 339-345.

16. Tsai YT, Liu JP, Tu YK, et al. (2012) Relationship between dietary patterns and serum uric acid concentrations among ethnic Chinese adults in Taiwan. Asia Pac J Clin Nutr 21, 263-270.

17. Song K, Du H, Zhang Q, et al. (2014) Serum immunoglobulin $\mathrm{M}$ concentration is positively related to metabolic syndrome in an adult population: Tianjin Chronic Low-Grade Systemic Inflammation and Health (TCLSIH) Cohort Study. PLOS ONE 9, e88701.

18. Austin PC (2011) An introduction to propensity score methods for reducing the effects of confounding in observational studies. Multivariate Behav Res 46, 399-424.

19. Jia Q, Xia Y, Zhang Q, et al. (2015) Dietary patterns are associated with prevalence of fatty liver disease in adults. Eur J Clin Nutr 69, 914-921.

20. Yang YX, Wang GY, Pan XC, et al. (2009) China Food Composition, 2nd ed. Beijing: Peking University Medical Press.

21. Zhang W, Doherty M, Pascual E, et al. (2006) EULAR evidence based recommendations for gout. Part I: Diagnosis. Report of a task force of the Standing Committee for International Clinical Studies Including Therapeutics (ESCISIT). Ann Rheum Dis 65, 1301-1311.

22. Alberti KG, Eckel RH, Grundy SM, et al. (2009) Harmonizing the metabolic syndrome: a joint interim statement of the International Diabetes Federation Task Force on Epidemiology and Prevention; National Heart, Lung, and Blood Institute; American Heart Association; World Heart Federation; International Atherosclerosis Society; and International Association for the Study of Obesity. Circulation 120, 1640-1645.

23. Craig CL, Marshall AL, Sjostrom M, et al. (2003) International physical activity questionnaire: 12-country reliability and validity. Med Sci Sports Exerc 35, 1381-1395.

24. McNeish D (2017) Exploratory factor analysis with small samples and missing data. J Pers Assess 99, 637-652.

25. Schlesinger N (2005) Dietary factors and hyperuricaemia. Curr Pharm Des 11, 4133-4138.

26. Ryu KA, Kang HH, Kim SY, et al. (2014) Comparison of nutrient intake and diet quality between hyperuricemia subjects and controls in Korea. Clin Nutr Res 3, 56-63.

27. Nakagawa T, Tuttle KR, Short RA, et al. (2005) Hypothesis: fructose-induced hyperuricemia as a causal mechanism for the epidemic of the metabolic syndrome. Nat Clin Pract Nephrol 1, 80-86.

28. Choi HK \& Curhan G (2008) Soft drinks, fructose consumption, and the risk of gout in men: prospective cohort study. BMJ 336, 309-312.

29. Nguyen S, Choi HK, Lustig RH, et al. (2009) Sugar-sweetened beverages, serum uric acid, and blood pressure in adolescents. J Pediatr 154, 807-813.

30. Chuang SY, Lee SC, Hsieh YT, et al. (2011) Trends in hyperuricemia and gout prevalence: Nutrition and Health Survey in Taiwan from 1993-1996 to 2005-2008. Asia Pac J Clin Nutr 20, 301-308.

31. D'Agostino RB Jr (1998) Propensity score methods for bias reduction in the comparison of a treatment to a nonrandomized control group. Stat Med 17, 2265-2281. 